\title{
How to help the statisticians correctly prepare maps
}

\author{
Maciej Zych ${ }^{\mathrm{a}}$ \\ a Statistics Poland, Spatial and Environmental Surveys Department, m.zych@stat.gov.pl
}

Keywords: Cartography handbook, Statistical maps, Thematic maps

\begin{abstract}
:
Statistics collects data in order to publish it after processing. Tables are the basic form of publishing statistical data and these tables include values of indicators selected to present specific phenomena. In the case of spatially related data (eg. data for countries, administrative units or other areas), maps may also be used for graphical presentation of this data. Unlike statistical tables or charts, statistical maps allow for simultaneous presentation of spatial distribution of the phenomenon and its value. Statistical maps are often supposed to lead to the synthesis of space characteristics, and not only to the analysis of individual places. These maps should organize the landscape of presented phenomenon and focus the readers' attention on main relations between data. Statistical maps allow for fast analysis and provide a quick overview of a general distribution of presented data and their values. They enable the presentation of spatially referred data in a more comprehensible, interesting and effective manner than tabular summaries and charts. For this reason, statistical maps are often used in the publications of statistical authorities.
\end{abstract}

The elaboration of statistical maps in statistical offices is usually done by the statisticians themselves. However, their professional education usually don't include a cartography training course. Therefore, they may not know all the rules governing the maps elaboration. This was the reason why in Polish public statistics it has become necessity to provide these people with the knowledge in cartography. The Polish-language manual Mapy statystyczne. Opracowanie $i$ prezentacja danych [Statistical maps. Data elaboration and presentation], which was published in 2017, was developed at Statistics Poland by Marek Pieniążek and Maciej Zych as an element of transferring this knowledge. Due to the interest in this publication also among foreign readers, in 2020 Statistics Poland published its modified English version (Figure 1).

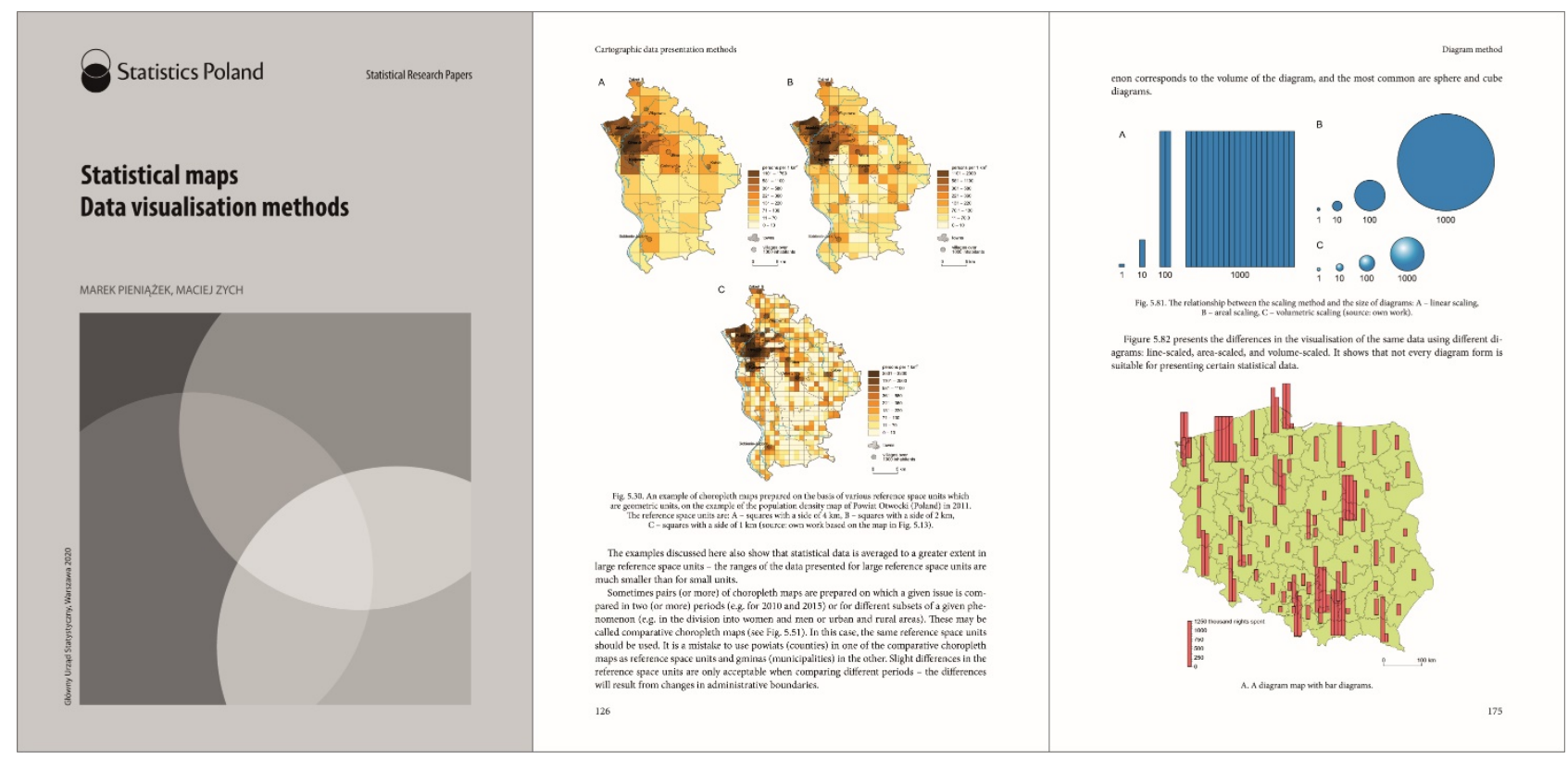

Figure 1. The cover of the handbook and examples of the content.

Poland-specific matters have been omitted, whereas certain topics not included in the Polish edition, that may be useful for an English speaking reader, have been added. The English edition was also supplemented with a number of changes and additions resulting from reviews and user comments on the first Polish edition.

The publication contains a number of procedures and solutions applied in preparing maps with the use of individual methods of cartographic presentation. These procedures and solutions often arise from map perception surveys, as well as research on the use of individual methods of cartographic presentation. They are not merely the authors' personal 
proposals, but rather constitute standards applied to the cartography as a whole. It is worth mentioning that the authors of the handbook applied consistent and logical division of the cartographic presentation methods used in Poland. It coincides with the divisions used in other countries, but differs from the ones used in the American and British literature, where the generally applicable systematics of cartographic presentation methods has not been established.

The handbook is richly illustrated with maps, mostly originally prepared for the Polish edition of the publication, or its English version - it is exceptional in the case of handbooks that most of the illustrations were made by authors specifically for the purposes of a given publication. The guide primarily discusses the correct and recommended solutions. However, due to popularity of certain incorrect practices, attention has also been drawn to them. Some of these incorrect solutions have been illustrated. In these cases, the drawing of a map is crossed out in red to clearly indicate that it is incorrect.

The handbook describes the rules for preparation of maps and not the tools (programs) used to create them. The most important thing is to know how to correctly create the map and choose a tool to do it properly, rather than adapt a map preparation method to a tool.

The publication begins with a chapter describing the elements of statistical maps. The types of content presented on maps, the graphic composition of a map, types of maps due to their graphic form, texts used on maps, map scale, map legend and map title are discussed here.

The next chapter is dedicated to the base map as an important element of statistical maps. The types of base maps and their sources, map projections and their selection, especially from the point of view of statistical maps as well as elements of generalization of a map content are described here.

The following two chapters discuss the elements of GIS: the principles of GIS, what types of data are used in it, how databases are built and the benefits of using metadata, how to store, organize and process the data. The basic elements of spatial analysis are also described here, such as attribute queries, intersecting features, union of them, merging, dissolving and determining buffers around features.

The most extensive chapter of the publication concerns the cartographic data presentation methods. Methods are discussed here in accordance with the division used by Lech Ratajski in book of 1989 Metodyka kartografii społeczno-gospodarczej [Methodology of socio-economic cartography], copied and modified by many subsequent authors. Ratajski divides the cartographic presentation methods into two qualitative and quantitative groups of methods, and then into individual methods. Due to the logical coherence of this division, it was used also in the English version of this publication. All the cartographic data presentation methods are described in hereby discussed cartography handbook, wherein the main emphasis is placed on quantitative methods that are used to present statistical data on maps. The dot method is discussed here - both the principles of preparing maps with this method, as well as its advantages and limitations in the presentation of statistical data. Another of the discussed methods is the isoline method - the emphasis is placed on isopleths here, i.e. theoretical isarithms, which are a variation of the isoline method that can be useful for elaboration maps based on statistical data.

The most extensive sub-chapter of this chapter concerns the choropleth method, which, apart from the diagram method, is most often used in presenting statistical data on maps. In the course of discussing this method, the emphasis is placed on the data that can be presented with this method. Moreover, very important issues of the reference space unit and determining classes are discussed here - a number of classes and methods for determining map classes (12 methods) are presented. Graphic schemes that can be used for choropleth maps are also characterized, as well as the correct elaboration of the legend for a map made using the choropleth method. Also 7 modifications of the choropleth method were characterized (some of them are sometimes considered separate methods).

The diagram method is also discussed extensively. The sub-chapter starts with the type of reference of diagrams diagrams related to an area or a point. Then, various types of diagrams used on maps elaborated with this method are characterized - i.e. diagrams showing the size of the phenomenon only, diagrams showing the structure of the phenomenon only, diagrams showing both the size and the structure of the phenomenon, dynamics diagrams, or comparative diagrams, as well as diagrams according to scaling methods: linear - bars, areal - e.g. circles, squares, volumetric - e.g. spheres, cubes. This sub-chapter also contains information on the correct placement of various types of diagrams on maps, their proper colors and shapes, and correctly elaborated map legend for maps prepared using the diagram method. 8 modifications of the diagram method are also characterized here, including linear diagram maps.

The last chapter provides guidelines for editing statistical maps and discusses the individual stages of map preparation, with particular emphasis on preparation in GIS software. The guide is addressed to all those involved in editing statistical and analytical publications, especially for the statisticians. 